\title{
Turbulent Flames with Compositionally Inhomogeneous Inlets: Resolved Measurements of Scalar Dissipation Rates
}

\author{
H. Cutcher ${ }^{\mathrm{a}^{*}}$, R.S. Barlow ${ }^{\mathrm{b}}$, G. Magnotti ${ }^{\mathrm{b}}$, A.R. Masri ${ }^{\mathrm{a}}$ \\ ${ }^{a}$ School of Aerospace, Mechanical and Mechatronic Engineering, The University of Sydney, \\ NSW, 2006 Australia. \\ ${ }^{b}$ Combustion Research Facility, Sandia National Laboratories, Livermore CA 94550, USA
}

* Corresponding author

Building J07, School of AMME

Faculty of Engineering and IT

University of Sydney, NSW 2006

Australia

Tel: + 61293513943

e-mail: hcut6115@uni.sydney.edu.au

\section{Preferred Colloquium: Turbulent Flames (5)}

\section{Word Count}

1. Intro 471

2. Experimental 800

3. Compositional Flame Structure 402

4. Scalar Dissipation 1592

5. Conclusion 237

6. Acknowledgements 73

7. References 419

subtotal 3994

Figure $1 \quad 137$

Figure $2 \quad 791$

Figure $3 \quad 820$

Figure $4 \quad 262$

Table $1 \quad 122$

Equation 123

Equation 246

subtotal 2201

Total Word Count $\quad 6195$ (Method 1)

Total Allowable Word Count 6200

Abstract Word count 212

Total allowable abstract word count $\quad 300$ 


\begin{abstract}
Highly resolved measurements of scalar dissipation rates in turbulent piloted $\mathrm{CH}_{4}$ /air flames with compositionally inhomogeneous inlets are presented. These were performed using Sandia's RamanRayleigh-LIF system but with data acquisition and processing strategies that result in enhanced spatial resolution and reduced noise. They complement earlier measurements with coarser resolution. The burner stabilising these flames enables variability in the mixture fraction profile at the exit plane. Earlier studies have shown enhanced stability at an optimal compositional inlet profile that leads to multiple modes of combustion, with premixed-stratified flames close to the jet exit but transitioning to diffusion-dominated burning downstream. It is found that at upstream locations, for jets with homogeneous inlets as well as for the high-temperature regions of flames with inhomogeneous inlets, both fine and coarse measurements of scalar dissipation rates yield similar results, giving confidence that measurements resolve the local dissipation scales. Downstream locations in homogeneous mixtures also show similar results for both coarse and fine measurements across all mixtures. Differences arise in the rich, inner regions of turbulent flames with inhomogeneous inlets, where the fine resolution measurements are more reliable due to the existence of steep gradients in composition. Both data sets provide a comprehensive platform to enhance the modelling of turbulent flames in the presence of multi-modes of combustion.
\end{abstract}

Keywords: Turbulent flames, scalar dissipation rates, partially premixed flames, inhomogeneous composition 


\section{Introduction}

The scalar dissipation rate, a highly relevant parameter in turbulent combustion modelling $[1,2]$ continues to challenge the combustion diagnostics community despite recent advances in laser imaging techniques $[3,4]$. The difficulty lies in the need to resolve the relevant dissipative flow structures yet maintain high signal to noise ratios (SNR) to enable a reliable measure of scalar gradients. There have been numerous attempts to measure scalar dissipation rates in turbulent flames [5-11], most with somewhat limited spatial resolution of $0.2-0.3 \mathrm{~mm}$, resulting in potentially significant spatial averaging affects. More recently, modification of the optical set-up at Sandia's Combustion Research Facility enabled multiscalar line measurements with $0.1 \mathrm{~mm}$ data spacing [12]. Advances in data acquisition and processing strategies [13] yielded data spacing of 20 microns, improved SNR, and spatial resolution approaching 60 microns. These techniques have been applied in the present measurements of turbulent piloted flames of methane with compositionally inhomogeneous inlets.

The burner, developed at the University of Sydney, enables variability in the mixture fraction profile issuing from the jet exit [14]. This is achieved by placing two concentric tubes within the pilot annulus, such that the inner tube can translate upstream of the burner exit. Varying the recess distance of the inner tube, varies the extent of mixing between fuel and air, leading to conditions not unlike those found in practical combustors. With fuel delivered through the inner tube and air through the outer, maximum flame stability was found to occur at some intermediate recess distance where compositional inhomogeneity is optimal [14-16]. Detailed measurements of species concentration and temperature at these conditions have revealed that fluid mixtures in the region adjacent to the hot pilot stream are generally close to stoichiometric, and this leads to additional heat 
release which augments flame stability $[15,16]$. Within the first 5 to 10 diameters from the jet exit plane a transition takes place from this mode of premixed-stratified combustion to diffusiondominated combustion.

These earlier measurements suffer from two drawbacks. First, estimates of turbulent length scales suggested that $0.1 \mathrm{~mm}$ resolution would be insufficient to measure scalar dissipation in the near field. Second, an insufficient number of radial scans were acquired at axial locations close to the jet exit, where significant changes in flame structure and the mode of combustion occur. The measurements reported here include more profiles within the near field, and they were taken using methods of spatial oversampling and wavelet denoising [13] that improve measurements of scalar gradients. The first section of the paper confirms key phenomena seen in the earlier measurements by showing selected scatter plots of temperature. The focus then shifts to scalar dissipation rates where the highly resolved measurements are compared with earlier measurements at lower resolution, analogous to a grid convergence test in simulations. New features of conditional scalar dissipation rates related to both flame conditions are highlighted.

\section{Experimental}

\subsection{The Burner:}

The Sydney piloted inhomogeneous burner, shown in Fig.1, is fully described elsewhere [14-16] and only brief details are given here. The inner tube ( $4 \mathrm{~mm}$ inner diameter, $0.25 \mathrm{~mm}$ wall thickness) can be recessed at a distance, $\mathrm{L}_{\mathrm{r}}$, within the outer tube $(7.5 \mathrm{~mm}$ inner diameter, $0.25 \mathrm{~mm}$ wall thickness). By varying the recession distance the degree of mixing can be controlled when fuel and air are delivered separately. For sufficiently large recess distances $\left(\mathrm{L}_{\mathrm{r}}>300 \mathrm{~mm}\right)$ the mixture is nearly homogeneous at the burner exit. For $\mathrm{L}_{\mathrm{r}}=0$ the flows are purely non-premixed. Intermediate recess distances lead to compositional inhomogeneity reflected in varying gradients in the mixture fraction profile at the exit plane. Maximum flame stability was noted for flames of methane at $\mathrm{L}_{\mathrm{r}}=75$ 
$\mathrm{mm}$ and for flames of compressed natural gas methane at $\mathrm{L}_{\mathrm{r}}=100 \mathrm{~mm}$, both with volumetric air/fuel ratio, $\mathrm{V}_{\mathrm{A}} / \mathrm{V}_{\mathrm{F}}=2.0$. The burner was located centrally in a $300 \mathrm{~mm} \times 300 \mathrm{~mm}$ wind tunnel giving a 15 $\mathrm{m} / \mathrm{s}$ uniform air co-flow.
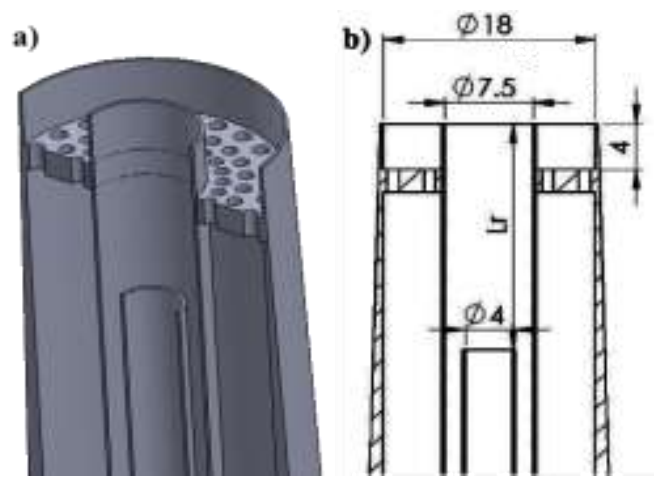

Fig. 1: Schematic (b) and isometric (a) view of modified Sydney piloted jet burner.

Five of the flames studied previously [15] are further investigated here, and relevant properties are listed in Table 1. FJ refers to cases with fuel $\left(99.97 \% \mathrm{CH}_{4}\right)$ issuing from the central tube (fuel in jet), while FA refers to fuel in the surrounding annulus and air issuing from the central tube. The volumetric air/fuel ratio of 2 is denoted by the '200' in the case codes. Three cases (FJ200-5GPLr300-59, FA200-5GP-Lr75-45, and FJ200-5GP-Lr75-80) were selected to have different inhomogeneity but the same departure from blow-off, a discussion of which is presented elsewhere in more depth $[15,16]$. The other two cases, FJ200-5GP-Lr75-57 and FJ200-5GP-Lr75-103, have the same inlet configuration as FJ200-5GP-Lr75-80 but different bulk jet velocities and different departures from blow-off. The bulk jet velocity, $U_{j}$, is calculated from the total volumetric flow rate at the exit plane.

A five gas pilot with a heat release rate of $2.22 \mathrm{~kW}$ was used for the cases presented here. It comprises of a mixture of $\mathrm{C}_{2} \mathrm{H}_{2}, \mathrm{H}_{2}$, air, $\mathrm{CO}_{2}$ and $\mathrm{N}_{2}$ in proportions to match the $\mathrm{C} / \mathrm{H}$ ratio and adiabatic temperature of a stoichiometric $\mathrm{CH}_{4} /$ air mixture, but with higher flame speed. This matched pilot is denoted by the ' $5 \mathrm{GP}$ ' in the case codes and distinguishes these flames from earlier work 
$[14,16]$, using a three gas pilot of $\mathrm{C}_{2} \mathrm{H}_{2}, \mathrm{H}_{2}$ and air, which had higher flame temperature and can be problematic for some modelling schemes. In this paper flames are sometimes referred to by excluding the '200-5GP', e.g. FA-Lr75-45, for simplicity.

Table 1: Selected Cases

\begin{tabular}{|c|c|c|c|c|c|c|}
\hline Cases & $\mathrm{L}_{\mathrm{r}}(\mathrm{mm})$ & $\mathrm{U}_{\mathrm{j}}(\mathrm{m} / \mathrm{s})$ & $\mathrm{U}_{\mathrm{bo}}(\mathrm{m} / \mathrm{s})$ & $\mathrm{U}_{\mathrm{j}} / \mathrm{U}_{\text {bo }}(\%)$ & $\mathrm{Re}_{\mathrm{j}}$ & $\mathrm{Hr}(\mathrm{kW})$ \\
\hline FA200-5GP-Lr75-45 & 75 & 45 & 64.5 & 70 & 21200 & 21.8 \\
\hline FJ200-5GP-Lr300-59 & 300 & 59 & 84.3 & 70 & 27600 & 28.5 \\
\hline FJ200-5GP-Lr75-57 & 75 & 57 & 114.3 & 50 & 26800 & 27.6 \\
\hline FJ200-5GP-Lr75-80 & 75 & 80 & 114.3 & 70 & 37500 & 38.6 \\
\hline FJ200-5GP-Lr75-103 & 75 & 103 & 114.3 & 90 & 48300 & 49.6 \\
\hline
\end{tabular}

\subsection{The Laser Diagnostics set-up:}

Raman and Rayleigh scattering, and laser induced fluorescence of $\mathrm{CO}$ at Sandia National Laboratories were used to provide single-shot profiles of temperature and major species $\left(\mathrm{O}_{2}, \mathrm{~N}_{2}\right.$, $\mathrm{CH}_{4}, \mathrm{H}_{2} \mathrm{O}, \mathrm{H}_{2}, \mathrm{CO}_{2}$ and $\mathrm{CO}$ ) across 6-mm segments in the flow. Multi-scalar diagnostic work performed on these $[15,16]$ and other turbulent partially-premixed flames $[6,17]$ has been expanded on by applying the technique of spatial oversampling and wavelet denoising described in [13]. Radial profiles at ten axial locations $(\mathrm{x} / \mathrm{D}=1,2,3,5,7,10,12,15,20$ and 30 , where locations $\mathrm{x} / \mathrm{D}=2,3$, and 7 correspond to added profiles in the present study) were obtained by traversing along the laser axis in 3-mm steps. 300-500 shots were taken at each step, giving 600-1000 samples at most locations. In addition, an increased number of samples (up to 3000) were acquired at regions of interests, identified in the previous campaign, to improve reliability of statistical analysis when combined with greater levels of conditioning. 
The current diagnostic setup has previously [11] been described in more detail but is explained briefly here. Raman and Rayleigh line imaging used four frequency doubled Nd:YAG lasers to achieve $\sim 1.8 \mathrm{~J} /$ pulse with a 400ns overall pulse duration. For the CO laser induced fluorescence measurements a $230.1 \mathrm{~nm}$ UV laser beam was used, aligned along the same axis as the Nd:YAG laser beams. Three CCD cameras were used to collect the line imaged Raman/Rayleigh/CO-LIF signals after passing through a pair of $150 \mathrm{~mm}$ achromatic lenses. The improved data acquisition and processing strategy involves spatial oversampling at a rate chosen to maximise signal to noise ratio and to take full advantage of the optical resolution [13]. The spatial resolution of the optical system is primarily limited to 60 microns by the Raman detection systems' line spread function [12] and cameras were binned over four pixels to collect across a 20 micron region. Wavelet denoising with an adaptive thresholding and reconstruction method was also applied as it was shown to give superior performance to other denoising algorithms [13]. With this approach the effective resolution of gradients in the reconstructed (smoothed) data, which are still represented at $20 \mu \mathrm{m}$ intervals, is not degraded by central differencing as it was for the earlier data which had $100 \mu \mathrm{m}$ data spacing.

\section{Compositional Flame Structure}

Figure 2 presents scatter plots of temperature, $T$, versus mixture fraction, $\xi$, for ten axial locations from $x / D=1$ to 30 . The vertical dashed line on each plot marks the stoichiometric mixture fraction. Results are shown for all five flames listed in Table 1, and these give insight into the development of combustion modes and the evolution of local extinction and re-ignition with increasing distance from the exit plane. While the qualitative features reported earlier [15] are reproduced here, subtle differences also exist due to the higher resolution of the present measurements. The following points are made: 
- For the three inhomogeneous cases (FJ-Lr75) near the exit plane, signs of premixed-stratified combustion can be seen in the near vertical rise from cold to hot of samples from across a range of mixtures within the flammable limits. These signs of premixed-stratified combustion diminish quickly, at a faster rate for lower velocities, and by $\mathrm{x} / \mathrm{D}=5$ most samples rising from room temperature are outside the rich flammable limit. The addition of measurements at $\mathrm{x} / \mathrm{D}=$ 2, 3 and 7 not included in previous work, allows better understanding of the transition away from premixed-stratified combustion in this region. The near-homogeneous cases (FA-Lr75 and FJ-Lr300) are characterised by a diffusion-dominated mode of combustion at all axial locations. From $\mathrm{x} / \mathrm{D} \approx 10$ the combustion mode of all flames has converged and differences are primarily driven by differences in velocity and departure from blow-off.

- Peak occurrence of local extinction occurs around $\mathrm{x} / \mathrm{D} 12$ in the FA-Lr75 flame, $\mathrm{x} / \mathrm{D}$ 15 in the FJ-Lr300 flame, and x/D 20 in the FJ-Lr75 flames. This is consistent with earlier results [15] and implies that regions of peak scalar dissipation rates occur at different locations in these flames. This aspect will be confirmed in the next section of this paper.

- Residual interference from $\mathrm{C}_{2}$ fluorescence can be seen at some locations, particularly in more homogeneous mixtures. While it is not possible to remove this completely through data processing methods, the effects of the remaining interference on the measured scalar dissipation rates reported in this paper are small, of similar levels to those seen in the earlier campaign [15].

- While results are shown here for temperature only, the results for species mass fractions are also similar across the two campaigns, and the higher resolution data allows a further look at key phenomena. The entire data will be posted on the web for access by modellers. 


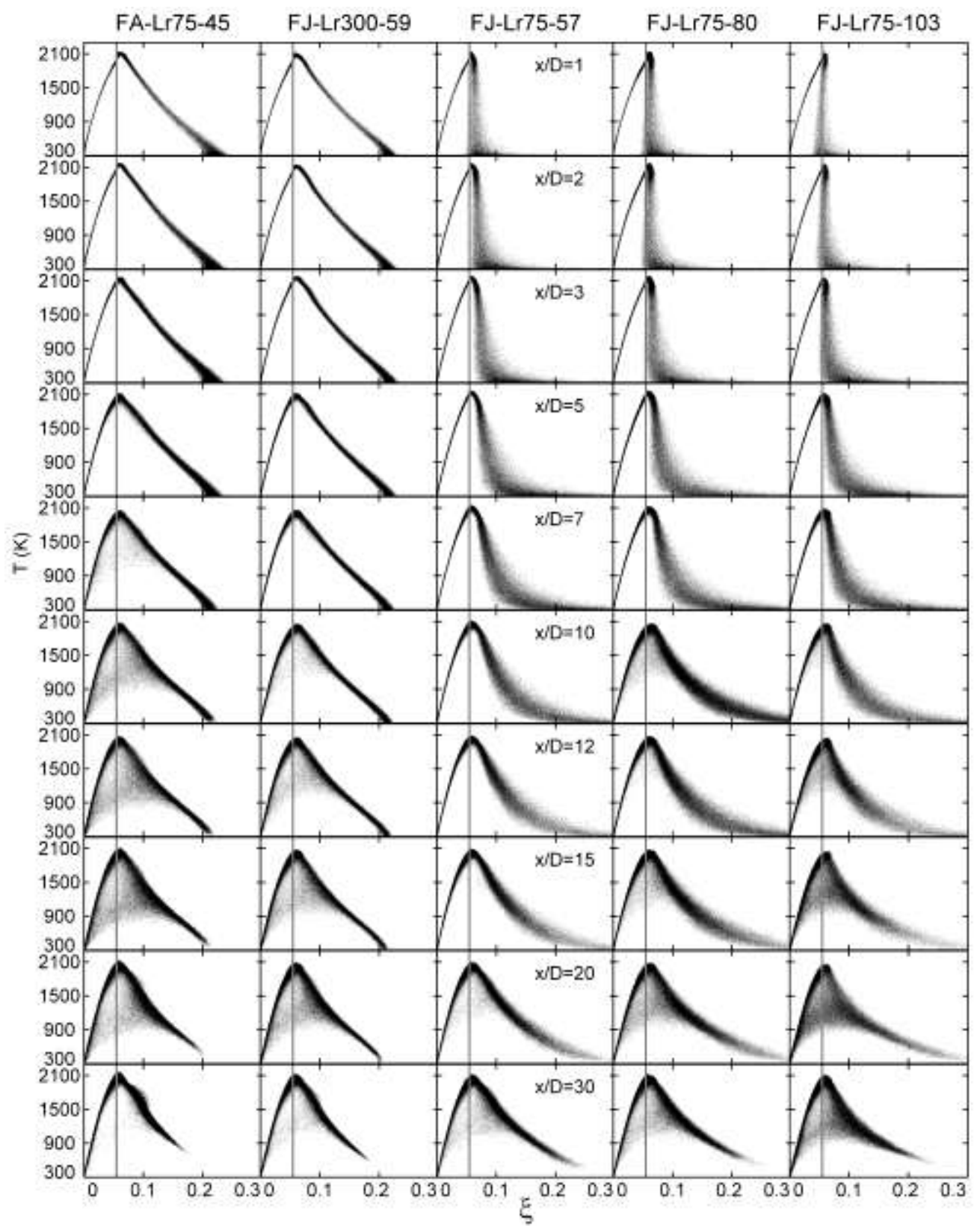

Fig. 2: Scatter plots of temperature in mixture fraction space. The vertical lines mark stoichiometric mixture fraction $\xi=0.055$.

\section{Scalar Dissipation Rates}

It is noted at the outset that all values of scalar dissipation reported here are one-dimensional, referring only to the radial contribution, $\chi_{\mathrm{r}}$, and evaluated using a central differencing scheme as per the following formula:

$$
\chi_{r}=2 D_{\xi}\left(\frac{\xi_{i+1}-\xi_{i-1}}{r_{i+1}-r_{i-1}}\right)^{2}
$$


with diffusivity evaluated using the following formula to fit laminar opposed flow calculations [10],

$$
D_{\xi}\left(\mathrm{cm}^{2} / \mathrm{s}\right)=-0.12013+0.74818(T / 1000)+1.1631(T / 1000)^{2}
$$

The application of a central differencing scheme to the $0.1 \mathrm{~mm}$ data from the previous experiments reduces the effective resolution of the scalar dissipation measurements but also reduces the impact of noise [18]. As mentioned in Section 2, central differencing has little impact on effective resolution or noise in the present wavelet denoised data. For convenience, the current high-resolution measurements are referred hereon with a symbol " $F$ " as per $\chi_{r, F}$, while the coarser data is labelled as $\chi_{r, C}$. The contributions of the other two dimensions to scalar dissipation may be inferred from earlier measurements [11] but are not likely to change the trends and relative magnitudes discussed in the remainder of this paper.

\subsection{Conditional Scalar Dissipation}

This section examines the scalar dissipation rate conditioned on mixture fraction and then identifies trends linked to differences in modes of combustion. Density weighted conditional mean profiles of $\chi_{r, F}$ and $\chi_{r, C}$ for axial locations of $\mathrm{x} / \mathrm{D}=1, \mathbf{2}, \mathbf{3}, 5,7,10,12,15$, and 30 can be seen in Fig. 3, with bold locations only having fine resolution data. The addition of data at $\mathrm{x} / \mathrm{D}=2,3$ and 7 allows an improved look at the effect of upstream mixtures on flame behaviour and the transition in combustion mode through this region. The mixture fraction range has been limited from 0 to the bulk jet mixture fraction $\xi=0.218$. This enables us to focus on key phenomena occurring in these regions. Note, as well, the differences in the y-axis scale between the plots. The following points are noted:

- The noise contribution to the measured scalar dissipation is minor for both resolutions, as can be seen from the near-zero levels at $x / D=1$ in the stoichiometric products of the premixed pilot for the FJ-Lr300-59 and FA-Lr75-45 flames. Fluctuations in the conditional mean profiles are 
mainly statistical; with scalar dissipation being highly intermittent, very large sample size would be required to achieve smooth profiles.

- A key feature of interest in the near-homogeneous cases (FA-Lr75, and FJ-Lr300) is the development of a twin-peaked structure which becomes more distinct with increasing distance from the jet exit plane. A local peak in conditional dissipation near $\xi=0.075$ is already apparent at $\mathrm{x} / \mathrm{D}=5$. $\mathrm{By} \mathrm{x} / \mathrm{D}=10$ the beginning of a local peak just to the lean side of stoichiometric can be seen. As this lean peak becomes more prominent with downstream distance, a local minimum develops near $\xi=0.06$, leading to the distinct twin-peak structure observed at $\mathrm{x} / \mathrm{D}=30$. These features and the changes with axial distance were also observed in the Sandia piloted flames $[10,11,19]$. Analysis of measured and simulated laminar diffusion flames [6,20] has identified a similar structure in scalar dissipation profiles and attributed it to the influence of differential diffusion on mixture fraction. It is clearly associated with a diffusion-dominated mode of combustion in the present near-homogeneous turbulent flames. Calculations for opposed flow laminar flames were performed using Chemkin (not presented here) for various mixtures ranging from lean to rich issuing against air and for differing strain rates. These are somewhat representative of the local conditions in the turbulent flames studied here and were found to show similar trends. As the equivalence ratio increases, a twin peaked structure also develops but becomes less distinct with increasing global strain rate. This is analogous to the effects of increasing bulk jet velocity in the turbulent inhomogeneous flames.

- Considering the three inhomogeneous flames (FJ-Lr75), at $\mathrm{x} / \mathrm{D}=10$, we can see the beginning of the localised peak just rich of stoichiometric in the $59 \mathrm{~m} / \mathrm{s}$ and $80 \mathrm{~m} / \mathrm{s}$ cases analogous to that clearly seen in the near-homogeneous cases. This peak is not visible until $\mathrm{x} / \mathrm{D}=30$ for the $103 \mathrm{~m} / \mathrm{s}$ flame, which is likely due to a combination of higher velocity, greater local extinction, and lesser influence of differential diffusion. The observed growth of a localised peak near $\xi=0.075$ in the 
inhomogeneous flames correlates with the previously reported $[15,16]$ region of transition of those flames from premixed-stratified to diffusion-dominated combustion. The fact that it exists in the near-homogeneous flames at all axial locations downstream of the pilot region but only appears farther downstream in the inhomogeneous cases corroborates the suggestion that it is an indicator of diffusion-dominated combustion.

- For axial locations up to and including $\mathrm{x} / \mathrm{D}=7$, conditional scalar dissipation is relatively low for mixtures near to stoichiometric in the inhomogeneous flames. Between $x / D=7$ and $x / D=10$, there is a significant increase in the dissipation rate especially in the two higher velocity cases. This increase corresponds with significant signs of local extinction, visible as low temperature samples, in Fig. 2. The low dissipation rates for mixtures near stoichiometric help explain the enhanced stability and delayed local extinction of these flames when compared to the homogeneous cases.

- The twin peaked structure including local minima at close to stoichiometric is close in structure to that observed in opposed flow [6] and homogeneous flames [21].

For the $\chi_{r, F}$ results in the inhomogeneous flames (FJ-Lr75), the peak scalar dissipation increases as the jet velocity increases and the flames approach blow-off. This is consistent with earlier results [15]. With increasing axial distance, the scalar dissipation rates gradually decreases in overall magnitude and is expected due to the shallower concentration gradients that form further downstream. 


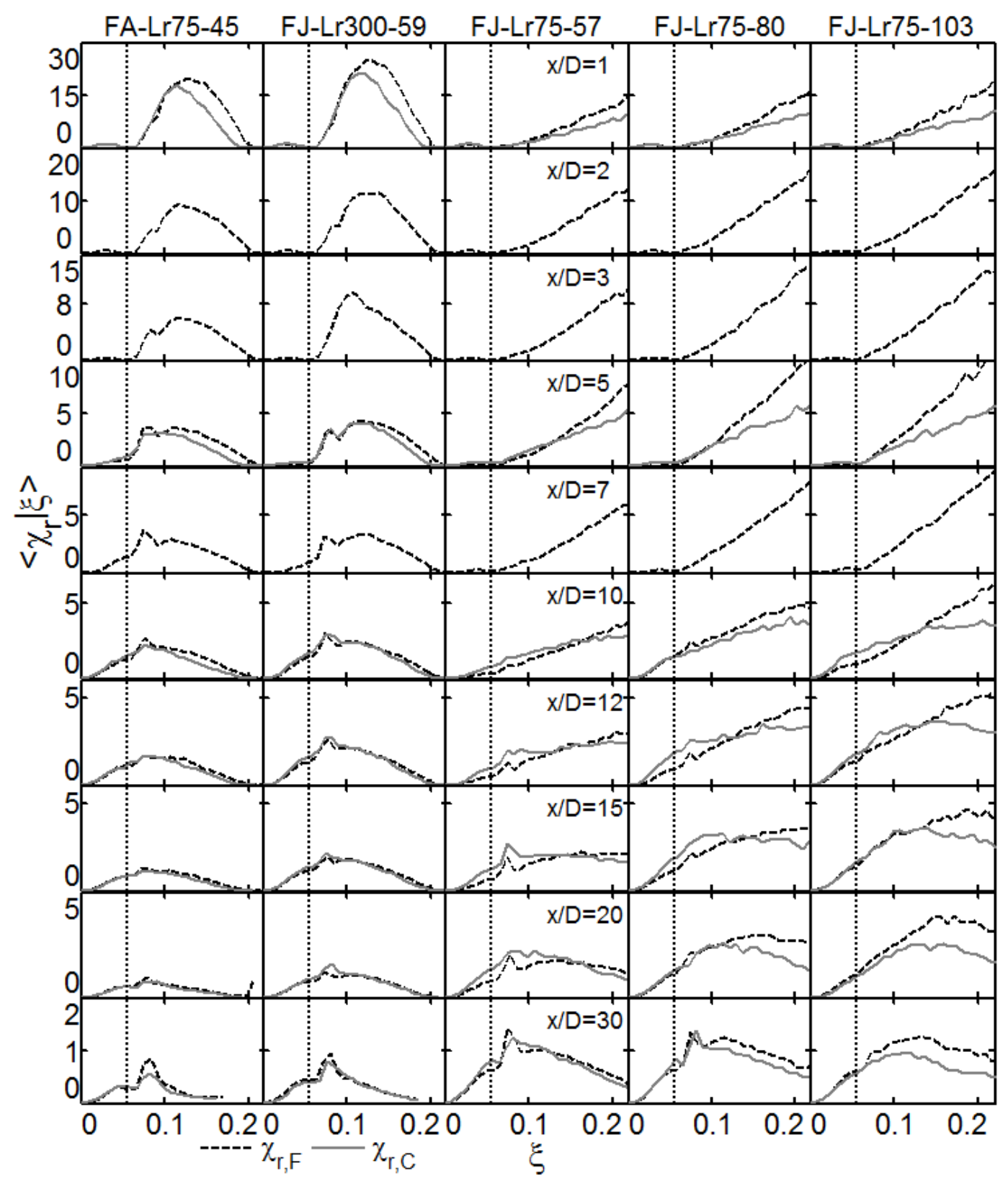

Fig. 3: Density weighted conditional mean profiles of scalar dissipation (radial contribution only) for a limited mixture fraction range, $0<\xi<0.22$. The profiles for fine and coarse resolutions are denoted by dashed and solid lines respectively. The vertical line marks the stoichiometric condition, $\xi=0.055$. Note the differing y axes scales at each axial location.

\subsection{Resolution Effects on $\chi_{\text {: }}$ :}

Observing Fig. 3 the conditional mean profiles for the fine (dashed lines) and coarse (continuous lines) data sets can be compared. The following points are made: 
- A significant result that was not expected is that the two resolutions show good agreement in the near field for lean and near-stoichiometric conditions, up to roughly $\xi=0.1$, which includes the main reaction zone for both types of flames. Based on measurements of thermal dissipation scales by high-resolution Rayleigh scattering in the near field of a non-piloted jet flame [22] and measurements of dissipation length scales in the Sandia piloted flames farther downstream [8], it was expected that the $0.1-\mathrm{mm}$ measurements would be insufficient to resolve mixture fraction dissipation in the near field reaction zones of the present flames. However, it appears that the presence of the laminar pilot flame significantly dampens turbulence in the developing shear layer at the edge of the jet and relaxes the dissipation scales. Heat release from premixedstratified combustion in the inhomogeneous cases would augment this effect. This agreement gives confidence that the present $\chi_{r, F}$ measurements are fully resolved in this critical region of evolution in the mode of combustion.

- At some intermediate axial locations in the inhomogeneous flames the $\chi_{r, F}$ results are lower than the $\chi_{r, C}$ results. This may be associated with somewhat lower levels of extinction in the present measurements compared to the previous measurements [15]. Differences in local extinction are attributed to the sensitivity of these flames, as they approach blow-off, to small variations in boundary conditions [3].

- For the near-homogeneous cases (FA-Lr75 and FJ-Lr300) there is a good similarity between $\chi_{r, F}$ and $\chi_{r, C}$ in terms of peak values and shapes of the conditional mean curves, except in rich conditions at locations near the exit plane. At these close locations, $x / D<10$, the scalar dissipation rate for richer mixtures is lower in the coarse data set, indicating that dissipation scales are under resolved in those conditions which correspond to the high-shear, lower temperature region of the jet. 
- The inhomogeneous cases (FJ-Lr75) show even greater differences between fine and course measurements, with the coarse resolution measurements significantly attenuating dissipation results in richer mixtures at all axial locations. The high dissipation levels revealed by the $\chi_{r, F}$ results correspond to regions of steep gradients in the Favre average radial profiles of mixture fraction [15] At upstream locations these high gradients and dissipation levels are found within the interior of the jet, separated for the burning region, so they would have little impact on flame behaviour.

- Differences between $\chi_{r, F}$ and $\chi_{r, C}$ increase as the jet velocity increases and the flames approach blow-off. This is clearly evident at $x / D=20$ and $x / D=30$ where the profiles of $\chi_{r, F}$ and $\chi_{r, C}$ almost overlap for case FJ-Lr75-57 but differ for the higher velocity cases FJ-Lr75-80 and FJ-Lr75-103. The higher shear rates in these flames lead to smaller structures which are harder to resolve.

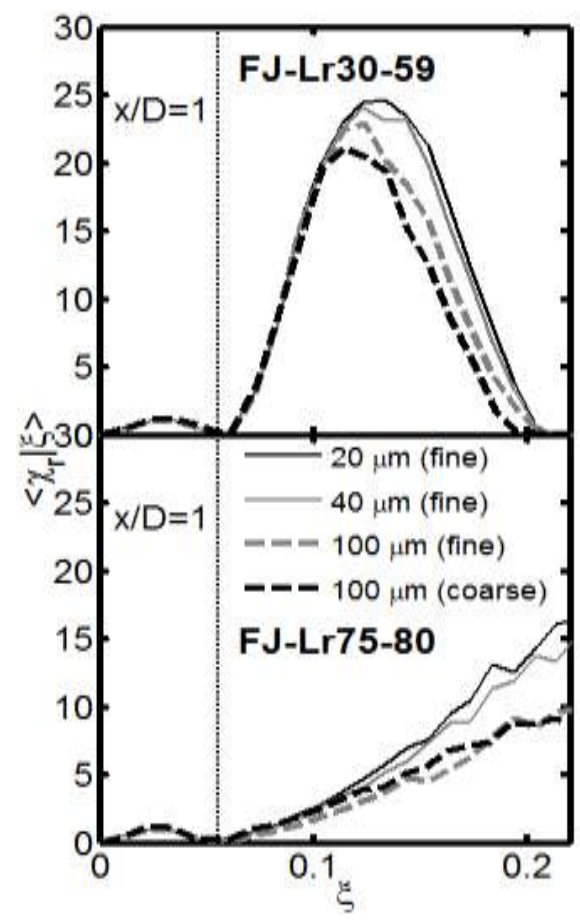

Fig. 4: Density weighted conditional mean scalar dissipation profiles for limited mixture fraction range, $0<\xi<0.22$. Pixels have been placed into 2 (grey, solid) and 5 (grey, dashed) wide bins and are compared with new and old data sets. 
Fig. 4 confirms the relative significance of resolution on the conditional mean profiles over differences in flame behaviour between the two campaigns and demonstrates that the new data is able to fully resolve scalar dissipation in all reacting regions of these flames. Density weighted conditional mean scalar dissipation profiles are shown for the fine and coarse data sets at $\mathrm{x} / \mathrm{D}=1$ alongside fine-resolution data that has been binned by two and five pixels to replicate 40 and 100 micron resolutions, respectively. The five pixel binned profiles were chosen to reproduce the original data set. Comparison shows that the coarse profile is well recovered when the resolution is matched especially for the inhomogeneous case suggesting the major of difference is a result of resolution rather than flame behaviour. A grid convergence test can be performed by comparing the three resolutions derived from the fine data set. Bins that are five pixels wide show significant difference but the profiles from two pixels wide bins are well converged with the unmodified data suggesting the resolution required to resolve scalar dissipation rates has been achieved. The resolution of smaller length scales has enabled the identification of physical phenomena related to multi-modes of combustion.

\section{Conclusions}

Two data sets with different spatial resolution are now available for turbulent flames stabilised on the modified Sydney piloted burner, and cases with inhomogeneous as well as homogeneous inlets are studied. The following conclusions are drawn:

- The trends observed in the compositional structure of the studied flames and the transition in combustion modes from premixed-stratified to diffusion-dominated are confirmed for both data sets.

- A local minimum located slightly rich of stoichiometric in conditional scalar dissipation profiles and a fuel-rich local maximum (near $\xi=0.075$ in the present flames) can be associated with differential diffusion effects in diffusion flames, and these were observed to develop as the 
inhomogeneous flames transitioned from premixed-stratified burning in the near field to diffusion-dominated combustion farther downstream.

- There are many regions and flame conditions where the scalar dissipation results are similar for the fine and coarse data sets. This includes downstream locations of cases with homogeneous inlets and high-temperature regions in the near field of flames with inhomogeneous inlets. This is comparable to a grid convergence test, providing confidence that dissipation is well resolved in those regions, without need for detailed knowledge of the local dissipation length scales.

- High local gradients existing in the rich interior of the inhomogeneous jet flames require the fine spatial resolution, even as far downstream as $\mathrm{x} / \mathrm{D}=30$. This evident from the differences between the coarse and fine scalar dissipation rates measured on the rich side of the FJ-Lr75 flames.

\section{Acknowledgements}

Work at the University of Sydney was supported by the Australian Research Council. Work at Sandia was supported by the Division of Chemical Sciences, Geosciences and Biosciences, Office of Basic Energy Sciences, US Department of Energy. Sandia National Laboratories is a multiprogram laboratory operated by Sandia Corporation, a Lockheed Martin Company, for the United States Department of Energy under contract DE-AC04-94-AL85000. Contributions by Bob Harmon in support of these experiments are gratefully acknowledged.

\section{References}

[1] S.B. Pope, Proc. Combust. Inst., 34, (1) (2013) 1-31.

[2] H. Pitsch, S. Fedotov, Combust. Theory Model., 5, (1) (2001) 41-57.

[3] R.S. Barlow, Proc. Combust. Inst., 31, (1) (2007) 49-75.

[4] V. Sick, Proc. Combust. Inst., 34, (2) (2013) 3509-3530.

[5] E. Effelsberg, N. Peters, Proc. Combust. Inst., 22, (1988) 693-700.

[6] D. Geyer, A. Kempf, A. Dreizler, J. Janicka, Proc. Combust. Inst., 30 I, (1) (2005) 681-689.

[7] S.P. Nandula, T.M. Brown, R.W. Pitz, Combust. Flame, 99, (3-4) (1994) 775-783. 
[8] G. Wang, A.N. Karpetis, R.S. Barlow, Combust. Flame, 148, (1-2) (2007) 62-75.

[9] R.S. Barlow, A.N. Karpetis, M.S. Mansour, A. Gomez, Proc. Combust. Inst., 30, (1) (2005) 673-680.

[10] A.N. Karpetis, R.S. Barlow, Proc. Combust. Inst., 29, (2002) 1929-1936.

[11] A.N. Karpetis, R.S. Barlow, Proc. Combust. Inst., 30, (1) (2005) 665-672.

[12] R.S. Barlow, G.-H. Wang, P. Anselmo-Filho, M.S. Sweeney, S. Hochgreb, Proc. Combust. Inst., 32, (1) (2009) 945-953.

[13] M.S. Sweeney, S. Hochgreb, M.J. Dunn, R.S. Barlow, Combust. Flame, 160, (2) (2013) 322 334.

[14] S. Meares, A.R. Masri, Combust. Flame, 161, (2) (2014) 484-495.

[15] R.S. Barlow, S. Meares, G. Magnotti, H. Cutcher, A.R. Masri, Combust. Flame, 162, (5) (2015) 3516-3540.

[16] S. Meares, V.N. Prasad, G. Magnotti, R.S. Barlow, A.R. Masri, Proc. Combust. Inst., 35, (2) (2014) 1477-1484.

[17] W. Meier, X.R. Duan, P. Weigand, Proc. Combust. Inst., 30, (1) (2005) 835-841.

[18] G.-H. Wang, N.T. Clemens, R.S. Barlow, P.L. Varghese, Meas. Sci. Technol., 18, (5) (2007) 1287-1303.

[19] R. Barlow, J. Frank, A. Karpetis, J. Chen, Combust. Flame, 143, (4) (2005) 433-449.

[20] Y.C. Chen, J.Y. Chen, Combust. Theory Model., 2, (4) (1998) 497-514.

[21] R.S. Barlow, A.N. Karpetis, Flow, Turbul. Combust., 72, (2004) 427-448.

[22] J.H. Frank, S.A. Kaiser, Exp. Fluids, 49, (2010) 823-837. 


\section{Table of Figures}

Fig. 1: Schematic (b) and isometric (a) view of modified Sydney piloted jet burner..... 4

Fig. 2: Scatter plots of temperature in mixture fraction space. The vertical lines mark stoichiometric mixture fraction $\xi=0.055$. 8

Fig. 3: Density weighted conditional mean profiles of scalar dissipation (radial contribution only) for a limited mixture fraction range, $0<\xi<0.22$. The profiles for fine and coarse resolutions are denoted by dashed and solid lines respectively. The vertical line marks the stoichiometric condition, $\xi=$ 0.055. Note the differing y axes scales at each axial location.

Fig. 4: Density weighted conditional mean scalar dissipation profiles for limited mixture fraction range, $0<\xi<0.22$. Pixels have been placed into 2 (grey, solid) and 5 (grey, dashed) wide bins and are compared with new and old data sets. The vertical line marks the stoichiometric condition, $\xi=0.055$. 